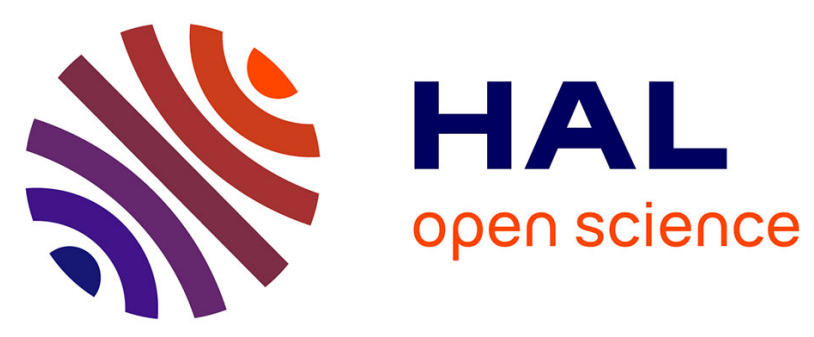

\title{
Perspective et point de vue
}

Alain Rabatel

\section{To cite this version:}

Alain Rabatel. Perspective et point de vue. Communications, 2009, 85, pp.23-35. halshs-00433003

\section{HAL Id: halshs-00433003 \\ https://shs.hal.science/halshs-00433003}

Submitted on 17 Nov 2009

HAL is a multi-disciplinary open access archive for the deposit and dissemination of scientific research documents, whether they are published or not. The documents may come from teaching and research institutions in France or abroad, or from public or private research centers.
L'archive ouverte pluridisciplinaire HAL, est destinée au dépôt et à la diffusion de documents scientifiques de niveau recherche, publiés ou non, émanant des établissements d'enseignement et de recherche français ou étrangers, des laboratoires publics ou privés. 


\section{Alain Rabatel \\ ICAR, UMR 5191, CNRS, Universités de Lyon 2 et Lyon 1 \\ Perspective et point de vue}

Si les notions de perspective et de point de vue (désormais PDV) ont un air de famille très accusé - dans le domaine des textes romanesques, auquel on bornera notre investigation , cela tient au fait qu'elles reposent partiellement sur des mécanismes linguistiques identiques et partagent une dimension énonciativo-pragmatique commune. Toutefois, les variations d'empan, plus amples avec la perspective, actualisant les mécanismes du PDV dans le cadre des textes et des genres, modifient la valeur des marques du PDV, accroissant leur esthétisation et leur poids interprétatif.

\section{Approches dictionnairiques de la perspective et du point de vue}

Dans les dictionnaires de langue, la perspective réfère à l'optique, au XIII ${ }^{\mathrm{e}}$ siècle, avant de se spécialiser en peinture à la Renaissance. La perspective est définie comme « une science qui enseigne à représenter les objets sur un plan, de la manière qu'ils paraissent à la vue, en gardant les distances et les situations » (Littré). Cette science de la perspectiva artificialis (par opposition $^{1}$ à la perspectiva naturalis) met à contribution des calculs savants pour rendre compte des objets selon le point d'où ils sont appréhendés et repose sur un certain nombre de techniques (perspective linéaire, jouant sur les lignes, perspective aérienne jouant sur le dégradé des couleurs). Dès le XVII $\mathrm{e}^{\mathrm{e}}$ siècle, le terme signifie également la «"manière dont on projette dans l'avenir le développement d'une situation actuelle" (1676), par exemple dans la locution "avoir quelque chose en perspective" (1688), "avoir en vue". Puis, à partir de cette expression, il désigne l'avenir prévisible (1689 : "une perspective agréable"), et telle manière particulière de voir les choses, d'interpréter le déroulement des événements (1757) » (Le Robert. Dictionnaire historique de la langue française). Au figuré, la perspective « se dit d'un événement éloigné mais possible» (Littré).

Deux points capitaux de dégagent des définitions précédentes. D'abord, la dimension intellectuelle du phénomène renvoie à l'expression ou à la construction d'une attente ou d'une intentionnalité (il n'a pas de perspective, avoir en vue), ou encore à l'expression d'une issue plutôt favorable (une heureuse perspective), présupposée par les expressions négatives (c'est sans perspective). Ensuite, le sens figuré pèse sur le sens de la notion en peinture et dans les autres arts : la perspective n'est plus seulement une science, c'est une technique au service d'une vision intentionnelle, théâtralisée, depuis un point élevé propice aux visions surplombantes. Certes, il n'existe pas que des perspectives surplombantes, et pourtant cette position et cette posture de surplomb ${ }^{2}$ correspondent à une quintessence de l'idée de perspective, dès lors que l'on pense le phénomène sans le rapporter à la peinture. Telle était la conception des moralistes classiques, illustrée par Bossuet dans son Sermon pour la deuxième semaine de Carème :

Le libertin inconsidéré s'écrie aussitôt qu'il n'y a point d'ordre : il dit en son cœur : "Il n'y a point de Dieu" [...] Mais arrêtez, malheureux, et ne précipitez pas votre jugement dans une affaire si importante. Peut-être que vous trouverez que ce qui semble confusion est un art caché ; et si vous savez rencontrer le point par où il faut

\footnotetext{
${ }^{1}$ C'est pourquoi, par métonymie, ainsi que le rappelle Le Robert. Dictionnaire historique de la langue française, une perspective est synonyme de toute représentation en trompe-l'œil, sur quelque support que ce soit. Cette métonymie est déjà présente dans le dictionnaire de Furetière.

${ }^{2}$ Le surplomb n'est pas seulement ni nécessairement un lieu élevé (une position haute) qui permet de voir loin et clair, c'est aussi une aptitude à dégager des grandes lignes, des enjeux, une posture intellectuelle ou un magistère moral.
} 
regarder les choses, toutes les inégalités se rectifieront, et vous ne verrez que sagesse où vous n'imaginiez que désordre.

Oui, oui, ce tableau a son point ; et le même Ecclésiaste, qui nous a découvert la confusion, nous mènera aussi à l'endroit par où nous contemplerons l'ordre du monde.

Quelle soit religieuse ou laïque, la perspective surplombante ressortit d'intérêts collectifs ou privés éprouvant un avantage à cette mise en spectacle qui est tout autant celle de l'objet que celle du sujet regardant.

Quant au point de vue, il correspond d'abord, selon les dictionnaires de langue, à la perspective en peinture, puis a pris le sens figuré de "manière de voir les choses" (1672), en particulier "opinion personnelle" (1673). Le composé se dit aussi d'une construction élevée dans un endroit bien situé (1763), sens qui a donné par métonymie celui d'"ensemble étendu qui sollicite le regard" (1763) » (Le Robert. Dictionnaire historique de la langue française). Littré définit le point de vue en une formule lapidaire («point sur lequel la vue se dirige ou s'arrête ») puis renvoie à " vue », avec les significations suivantes : " faculté de voir, fin que l'on se propose, projet, manière de voir, opinion, aperçu, idée ». Littré privilégie le complément (la vue), en traitant la tête nominale de l'expression, (le point), comme une question purement technique.

Or, si l'on quitte le terrain des définitions dictionnairiques pour aborder, sous un angle scientifique, cette fois, la question du PDV, le «point» est décisif, dans son ambivalence 3 , parce qu'il concerne le sujet regardant et qu'il est impossible de traiter de la question de la représentation des objets en faisant l'impasse sur les interactions entre l'objet et le sujet. Ces interactions sont sous-estimées dans la définition de Littré, mais aussi, d'une certaine façon, dans les approches techniques de la perspective, reposant sur des calculs et des techniques qui, par leur caractère « scientifique », réduisent l'observateur (interchangeable) à un point (le « point de l'œil », ou « point du sujet », cf. Damisch, 1987, Comar, 1992 : 35), comme si seul comptait l'emplacement d'où les choses sont vues, et comme si, donc, il était possible de faire abstraction de la personne du spectateur : on a beau voir du même point, on ne voit malgré tout pas les mêmes choses, sauf à se limiter aux distances et à la position des objets. Bref, et c'est là l'essentiel, le point de vue, du fait de la polysémie de vue, déborde de part en part la dimension « scientifique » de la perspective ${ }^{4}$.

La notion, telle du moins qu'elle est théorisée dans les études narratologiques ou linguistiques, a un substrat théorique sous-jacent beaucoup plus riche : voir, représenter, faire voir/penser, depuis un certain point qui organise la vue et influe sur l'impression que cette vue doit susciter sur le destinataire. Cette dimension pragmatico-argumentative de la vue explique que celle-ci ne soit pas la reproduction vériste du réel, mais une re-présentation, une mise en spectacle qui oblige à donner à la vue tout son empan, c'est-à-dire à prendre en compte que le fait que toute perception manifeste, d'une façon ou d'une autre, des opinions qui correspondent à des manières de voir ${ }^{5}$. Si l'on résume en élargissant à tous les domaines artistiques les tensions entre perspective et PDV en peinture, on gardera tout d'abord à l'esprit

\footnotetext{
${ }^{3}$ Cf. supra, la distinction entre position et posture.

${ }^{4} \mathrm{~S}$ 'en tenir à la simple « saisie géométrique de l'espace visible », c'est adopter « une perspective de cyclope » (Comar, 1992 : 36), bien nommée car l'expérience fondatrice de Brunelleschi repose sur la vision à travers un œeilleton creusé dans une planchette. Léonard de Vinci recommandait de corriger la perspective en intégrant la vision binoculaire, la variabilité des PDV (apud Hamou, 1995 : 33). A plusieurs reprises, notamment durant la guerre de la perspective à l'Académie de peinture entre 1650 et 1670, le débat a opposé les tenants d'une stricte orthodoxie géométrique (Abraham Bosse) et les partisans d'une adaptation (Le Brun), (ibid. : 32-33 et 221-338). Bref, la remise en cause de l'illusionnisme perspectif, en se basant sur la complexité de l'expérience visuelle, ne date pas que du livre mémorable de Panofski (1932), elle est aussi en germe chez Descartes, pour qui l'expérience sensible a toujours lieu en situation (Hamou, $1995: 43-44$ ).

${ }^{5}$ Le Dictionnaire d'analyse du discours (Charaudeau et Maingueneau, 2002) et les Termes et concepts pour l'analyse du discours (Détrie, Siblot, Verine, 2001) développent des analyses similaires.
} 
qu'en peinture, la perspective, en tant que problématique générale, englobe le point de vue, comme une des techniques contributives; ensuite, que le point de vue se scinde lui-même en points différents - le point de fuite n'est pas le point de l'œil - ; enfin, que la perspective, si on dépasse toutes les techniques qui viennent à son appui (tels les perspectographes de Dürer, panneau de verre, portillon, treillis, etc.) est un art des proportions qui ne peut pas ne pas rencontrer la question de la relativité du point de vue.

\section{Conceptions restreintes ou étendues du point de vue}

Cette conception est au cœur de notre approche des perceptions, dans le cadre du PDV représenté (Rabatel, 1997 et 1998). L'approche énonciativo-pragmatique met en relief le dialogisme des perceptions: le choix des dénominations, des qualifications, du procès de perception indiquent toujours-déjà un point de vue et un savoir sur l'objet de discours :

(1) Gilbert, qui depuis de longues années n'avait pas vu le roi, examinait en silence cet homme que Dieu avait donné pour pilote à la France, au moment que la plus rude tempête que la France eût encore à subir.

Ce corps gros et court, sans ressort et sans majesté, cette tête molle de formes et stérile d'expression, cette jeunesse pâle aux prises avec une vieillesse anticipée, cette lutte inégale d'une matière puissante contre une intelligence médiocre, à laquelle l'orgueil du rang donnait seul une valeur intermittente, tout cela, pour le physionomiste qui avait étudié avec Lavater, pour le magnétiseur qui avait lu dans l'avenir avec Balsamo, pour le philosophe qui avait rêvé avec Jean-Jacques, pour le voyageur enfin qui avait passé en revue toutes les races humaines, tout cela signifiait dégénérescence, abâtardissement, impuissance, ruine. (Dumas, Ange Pitou Laffont $1990: 845)$

Même si c'est le narrateur qui raconte et que Gilbert ne « dit » rien, la narration épouse son PDV, tentant de déterminer à travers des signes physiques significatifs l'état moral du roi. Les nombreux démonstratifs correspondent à une mise en relief de phénomènes saisis intentionnellement. Il ne s'agit pas de référer à une " tête molle » dans l'absolu, mais à ce que ce symptôme, qui saute aux yeux, signifie pour le physionomiste, le magnétiseur ou le philosophe qu'est Gilbert, en sorte que les démonstratifs indiquent un aller et retour rapide entre une observation objective de traits et un masse de données qui permettent de sélectionner et interpréter les informations significatives. Bref, la description n'a pas seulement une visée informative, elle a surtout une visée argumentative.

La problématique du PDV concerne notamment les perceptions, les paroles (PDV représenté, raconté, asserté, cf. Rabatel, 2004a), mais elle n'a pas à être bornée a priori tel ou tel domaine sémantique, puisque la référenciation des énoncés n'est jamais neutre, et construit toujours une certaine manière de voir pour le destinataire, ainsi que le dit Ducrot :

Le locuteur, responsable de l'énoncé, donne existence, au moyen de celui-ci, à des énonciateurs dont il organise les points de vue et les attitudes. Et sa position propre peut se manifester soit parce qu'il s'assimile à tel ou tel des énonciateurs, en le prenant pour représentant (l'énonciateur est alors actualisé), soit simplement parce qu'il a choisi de les faire apparaître et que leur apparition reste significative, même s'il ne s'assimile pas à eux (Ducrot, $1984: 205)$

J'appelle 'énonciateurs' ces êtres qui sont censés s'exprimer à travers l'énonciation, sans que pour autant on leur attribue des mots précis ; s'ils 'parlent', c'est seulement en ce sens que l'énonciation est vue comme exprimant leur point de vue, leur position, leur attitude, mais non pas, au sens matériel du terme, leurs paroles (Ducrot, $1984: 204)$

Le PDV correspond à la façon dont un sujet envisage un objet, à tous les sens du terme envisager, que l'objet soit un objet concret ou un objet langagier. Quant au sujet, responsable de la référenciation de l'objet, il exprime son PDV soit directement, par des commentaires explicites, soit indirectement, par la référenciation, c'est-à-dire à travers les choix de sélection, de combinaison, d'actualisation du matériau linguistique, et ce dans tous les cas de 
figure, depuis les choix les plus subjectifs aux choix apparemment les plus objectivants, depuis les marques les plus explicites aux indices les plus implicites.

La source du PDV est donc un énonciateur (Ducrot, 1984, Rabatel, 1998), autrement nommé foyer (Genette 1972, 1983), focalisateur (Bal, 1977), sujet de conscience (Zribi-Hertz, 1990, Banfield, 1995), foyer d'empathisation (Kuno, 1976, Forest, 2003), centre de perspective (Lintvelt, 1981, Rabatel, 1997), etc. La convergence des approches, au-delà des différences de dénomination et de cadres théoriques de référence, souligne le caractère radicalement dialogique du PDV, ramené à une instance qui peut se trouver coïncider avec le locuteur (il y a alors syncrétisme entre le locuteur et l'énonciateur, et le locuteur exprime dans et par son discours un PDV qui est le sien), ou qui, le plus souvent, ne coïncide pas avec le locuteur, comme dans les cas de discours rapportés, et, d'une façon générale, dans les phénomènes de mention, donc dans les phénomènes d'ironie, dans les PDV narratifs, où le locuteur exprime dans et par son discours des PDV qui sont ceux d'un autre (allocutaire, tiers, doxa, personnage, etc. $)^{6}$.

Partant de là, tout énoncé indique nécessairement le PDV de son énonciateur : et l'on serait tenté de dire qu'il en va du PDV comme de l'engagement chez Sartre : de même que le sentiment intime du non engagement n'exonère pas la personne de son engagement objectif, le fait de n'avoir pas de PDV personnel témoigne malgré tout d'un PDV, fût-ce un PDV par défaut, qui est souvent le PDV naturalisé de la doxa ambiante. Les PDV doxiques dans lesquels un sujet ne fait que reproduire le PDV du groupe traduit les intentions de ce groupe auxquelles l'énonciateur adhère sans discussion. Tout PDV qui s'illusionne (c'est le propre de l'idéologie), tout PDV qui a pour source des individus agis, dominés, ballottés par l'histoire porte néanmoins la trace d'une intentionnalité, à l'instar de l'exemple ci-dessous :

(2) Pour nous autres, un soldat français, cela devait combattre, vaincre ou périr. Sans autre choix. On nous l'avait enseigné à l'école primaire. Nous avons tous été gavés au clairon. D'avoir trop soufflé dedans, il nous est resté un goût de cuivre aux lèvres... Vercingétorix... Debout les morts !... Le petit Bara... Marchons, marchons !... Les marins du Vengeur... On les aura! Turenne... J'y suis j'y reste !... Qu'un sanguimpur... Le petit Tondu... Austerlitz, Marengo, Wagram, Iéna... A moi Auvergne !... Du haut de ces pyramides... Duguesclin, Bayard, Saint Louis, Jeanne d'Arc... Couper cabêche... Tirez les premiers !... Abreuve nos sillons, tas d'cochons !... La charge de Reichshoffen... Y'a bon !... Madelon ! Madelon! Madelon !... Le zouave du pont de l'Alma... Plutôt mourir que de se rendre !... La monteras-tu la côte... Le dernier carré... Les dernières cartouches.... L'as-tu vu, la casquette, la casquette ?... La tranchée des baïonnettes... Pan! Pan! L'arbi... « Merde !» répondit Cambronne...

On possédait son histoire de France.

Nous savions ce qu'il nous fallait faire. Pas difficile. Je ne veux pas dire qu'on avait exagérément envie de se battre, non. Je veux dire simplement qu'on n'a pas envisagé une seule fois de se rendre. Pourtant, on s'est rendus, et sans y mettre trop de façons ; il faut le reconnaître. (Calet, Le bouquet Gallimard 1947, 2000 : 13-14)

Bref, le PDV est une catégorie linguistique transversale (Rabatel, 2005), dialogique et à dimension argumentative, dont on trouve des traces à toutes les époques, même si ces traces prennent des formes différentes. C'est précisément par le biais de ces pratiques discursives situées, que le PDV s'articule avec la perspective.

\section{Pour une approche continuiste des relations entre point de vue et perspective}

Est-on fondé à conclure que, dans les pratiques artistiques langagières, PDV et perspective traitent des mêmes objets, selon des cadres théoriques différents, ou

\footnotetext{
${ }^{6}$ A charge pour le locuteur de préciser sa position par rapport au PDV de l'énonciateur, s'il n'y pas coïncidence. C'est ainsi que dans l'exemple (1), le narrateur, du fait même qu'il ne manifeste pas de distance, et compte tenu du pacte de lecture réaliste, entérine par défaut le PDV de Gilbert. En (2), en revanche, par ses commentaires explicites, le narrateur n'entérine pas les points de vue doxiques.
} 
correspondent à des cadres théoriques largement similaires, mais qui rendent compte d'objets distincts?

Il est vrai que les cadres théoriques sont très proches, à preuve l'interchangeabilité des termes focalisateur, énonciateur, centre de perspective, pour renvoyer à une même notion. Certes, on peut objecter un laxisme philologique et/ou un éclectisme notionnel, mais on peut reconnaître là, plus sûrement, la trace cumulative des diverses théories qui ont traité de la question. L'hypothèse peut être étayée de façon plus consistante par l'existence de caractéristiques communes aux deux notions, qui ne se laissent pas réduire à des définitions normatives réductrices du type de celles qui opposeraient un PDV inintentionnel, nonhiérarchique, non surplombant à une perspective intentionnelle, hiérarchique et surplombante.

Ces dichotomies sont discutables, parce qu'elles érigent en norme des pratiques qui sont démenties par la réalité des usages. L'opposition entre perspective surplombante et PDV non surplombant est commode, mais de convenance, tant il est vrai qu'il existe des PDV surplombants en langue, comme le manifestent les phénomènes de surénonciation (Rabatel, 2004b) : en (2), le locuteur/énonciateur premier, qui rapporte ironiquement, en les accumulant et en les déformant, les PDV des énonciateurs seconds exprimant la bravoure et le panache français, fait ainsi entendre une dissonance qui trouve sa conclusion dans la pointe finale. Evidemment, on pourrait tirer prétexte de l'exemple pour opposer la perspective surplombante du narrateur au PDV dominé des personnages. Mais cette analyse est discutable, car il existe des perspectives auctoriales qui ne sont pas surplombantes, et des perspectives actoriales qui le sont (Rabatel, 1997).

De même, le fait que la perspective correspond souvent à des vues ordonnées à partir d'un centre de perspective unique ne doit pas être généralisé, comme il appert avec les ruptures volontaires de perspective dans la peinture cubiste ou avec la polyphonie des romans dostoïevskiens. De plus, la notion de hiérarchie est complexe et le PDV, dès lors qu'il repose sur au moins deux propositions, hiérarchise les PDV, avec un PDV principal et un PDV subordonné (Rabatel, 2003). C'est ce qui se passe dans l'exemple précédent, puisque tous les énoncés initiaux relatifs à la bravoure française abondent le point de vue hiérarchique du peuple français invincible, point de vue lui-même subordonné au fait que ce peuple brave et bravache s'est « pourtant rendu, et sans y mettre trop de façons. »

En revanche, il est possible de considérer que le PDV et la perspective correspondent à deux approches similaires portant sur des objets distincts, sans se superposer totalement, selon une optique continuiste. Ainsi, PDV et perspective relèvent d'une même problématique pragmatique (faire voir, faire penser) qui joue dans des cadres différents et qui acquiert de ce fait des valeurs spécifiques.

Les caractéristiques linguistiques du PDV, en étant actualisées dans des textes, euxmêmes inscrits dans des genres et des époques, participent à la construction des perspectives. L'actualisation dynamique des catégories linguistiques dans le cadre des discours et des genres dote le PDV d'une valeur et d'une expressivité redoublées du fait de la clôture des œuvres et de l'objectivation de traits de style génériques ou spécifiques. La clôture aide à penser une totalité, en distinguant l'intérieur d'avec l'extérieur, tout en rendant plus lisibles ses structures internes. Cette dialectique existe aussi dans d'autres sémiotiques: les perspectives picturales ${ }^{7}$, architecturales, urbanistiques ou paysagères requièrent une clôture d'avec leur environnement pour faire sens et système à l'intérieur de l'espace ainsi délimité et organisé. Et il n'est pas douteux que les genres eux-mêmes correspondent à une certaine forme de clôture, du fait de leur structure.

Les mêmes phénomènes sont tantôt appréhendés à partir d'un contexte étroit, tantôt à partir d'un contexte plus large. Ainsi, les traits linguistiques du PDV qui acquièrent une

\footnotetext{
7 On trouve un équivalent de la clôture dans l'importance du tracé perspectif chez Filarète, dans son Traité d'architecture (1460-1464), cf. Hamou, $1995: 88-89$.
} 
signification à la fois plus ample plus profonde, dans les genres de discours littéraires, dans des œuvres appréhendées comme un tout. On considèrera donc que les points de vue partiels de tel personnage, sur un autre (Fabrice sur la Sanseverina), sur un lieu ou un événement (Fabrice depuis sa prison, Fabrice à Waterloo) construisent la perspective actorielle, tout comme les PDV ponctuels du narrateur se cumulent pour former la perspective auctorielle. Ce sont bien les PDV, au sens énonciatif/pragmatique, qui construisent les perspectives dominantes des œuvres ${ }^{8}$.

Et du même coup, les déséquilibres qui affectent les premiers bouleversent les dernières. Mais, avant d'envisager ces évolutions, il convient d'insister sur un point décisif. Si PDV et perspective gagnent à être envisagés dans une optique continuiste, ils ne doivent pas être considérés comme deux réalités totalement superposables : de même qu'en peinture, le point de vue est une technique parmi d'autres au service de la perspective, le PDV, tel que nous l'avons défini dans notre deuxième partie n'est qu'une des techniques qui construisent la perspective.

\section{Les évolutions de la perspective : de la fin des valeurs et des certitudes à l'émergence d'un monde complexe et multipolaire et des parcours de lecture}

Conclure qu'avec l'introduction de la perspective à la Renaissance la vision du monde s'en trouve bouleversée, tout comme la place qu'y tenait Dieu est à la fois très vrai, pour notre culture européenne, mais ne peut être généralisé sans plus de précaution, sauf à verser dans un européanocentrisme lui-même réducteur ${ }^{9}$.

Le passage d'une perspective surplombante (regard de Dieu, regard totalisant/totalitaire d'une pensée érigée en dogme, comme dans le roman à thèse) a progressivement laissé la place aux œuvres qui ont contesté la pertinence d'un principe unique d'organisation et d'explication du monde et d'une manière de raconter à partir d'un centre unique surplombant (à l'instar du narrateur hugolien), ou irradiant en créant des personnages dotés d'une volonté de puissance similaire à l'énergie du narrateur (Balzac). Listons quelques unes des techniques qui, à côté du PDV, ont contribué aux remises en question de la perspective surplombante : multiplication et autonomisation croissante des personnages, remise en cause du personnage principal, travail de sape sur les représentation des actions, des paroles ou des pensées, techniques de contrepoint, absence de hiérarchisation entre personnages et systèmes de valeur antagonistes du fait de la discrétion de la voix narratoriale, intensification de la remise en cause des repères en accroissant les phénomènes de récursivité, de ratiocination (monologue intérieur), au point de brouiller les frontières entre le monde pensé et le monde réel (Raimond, 1968).

Mais cet affaiblissement de la perspective surplombante n'est ni homogène, ni unidirectionnel, sauf à évacuer l'immense cohorte des œuvres qui n'entrent pas dans ce schéma. De plus, les modifications de la perspective ne se réduisent pas à la fin des repères et des principes uniques d'explication du monde ou à l'« ère du soupçon », à la crise des valeurs (Chartier, 1990, Reuter, 1991); elles témoignent aussi de l'émergence d'une pensée complexe, d'une représentation complexe du monde comme des parcours de lecture et d'interprétation.

C'est ce qu'indiquent les évolutions de la fonction auteur qui rejaillissent sur la perspective auctoriale. Barthes soulignait combien la représentation de la fonction auteur, en littérature, correspond à une sorte d'arrêt sur image qui ne correspond qu'aux quelques siècles

\footnotetext{
${ }^{8}$ Ces relations PDV-perspective se retrouvent dans d'autres types de texte : dans les textes argumentatifs, par exemple, les relations PDV-perspective sont homologues des relations argument-thèse.

${ }^{9}$ Car la perspective européenne se réduit pas à la perspective centrale, la perspective parallèle existe aussi en Europe ; il en va différemment pour la perspective en éventail, en usage dans le Moyen- ou l'Extrême-Orient.
} 
qui ont suivi l'invention de l'imprimerie et l'avènement de la Renaissance. Auparavant, et notamment durant le long Moyen Âge,

ce que nous appelons l'auteur n'existe pas ; autour du texte ancien, seul texte pratiqué et en quelque sorte géré, comme un capital reconduit, il y a des fonctions différentes : 1) le scriptor recopie purement et simplement ; 2) le compilator ajoute à ce qu'il copie, mais jamais rien qui vienne de lui-même ; 3) le commentator s'introduit bien dans le texte recopié, mais seulement pour le rendre intelligible ; 4) l'auctor, enfin, donne ses propres idées mais toujours en s'appuyant sur d'autres autorités. Ces fonctions ne sont pas nettement hiérarchisées : le commentator, par exemple, peut avoir le prestige qu'aurait aujourd'hui un grand écrivain [...]. Ce que par anachronisme nous pourrions appeler l'écrivain est donc essentiellement au moyen âge : 1) un transmetteur : il reconduit une matière absolue qui est le trésor antique, source d'autorité ; 2) un combinateur : il a le droit de 'casser' les œuvres passées, par une analyse sans frein, et de les recomposer (la 'création', valeur moderne, si l'on en avait eu l'idée au moyen âge, y aurait été désacralisée au profit de la structuration). (Barthes, 1970 : 184 185)

L'image de l'auteur née à la Renaissance est remise en cause dès la fin du XIX ${ }^{\mathrm{e}}$ siècle, d'abord dans les sciences exactes, où il est en effet difficile de circonscrire exactement la part de tel ou tel dans une découverte, une invention ou une innovation, puis dans les sciences humaines, ainsi que le rappelle Foucault dans «Qu'est-ce qu'un auteur? » (1969, 2001a). La figure de l'auteur prend ses distances avec l'image traditionnelle de l'auteur-démiurge, au point qu'il devient quasiment impossible de l'appréhender à partir d'un sujet originaire unique Foucault, 2001a: 543, 2001b : 38-39). Ces évolutions affectent le domaine artistique, non seulement en raison de l'existence des interdiscours et des phénomènes d'intertextualité, mais encore du fait de la montée en puissance de nouvelles technologies (structures hypertextuelles, écritures coopératives ou interactives où dominent les notions de parcours et de négociations/construction du sens). De même, l'extension de nouveaux rapports de production avant, pendant et après le processus créateur, obligent l'écrivain (ou les personnes qui assument collectivement ce rôle, comme dans l'écriture d'un scénario, d'un article ou d'un livre) à intégrer des attentes, des commandes diverses, plus ou moins nettement objectivées dans un cahier des charges qui, sans faire disparaître le processus créateur, en modifie les contours et les enjeux.

«Il n’y a qu'un point invisible, écrivait Pascal, qui soit le véritable lieu. [...] La perspective l'assigne dans l'art de la peinture, mais dans la vérité et dans la morale qui l'assignera ? (Pascal, Pensées, Lafuma, 21). Or, dès lors qu'il n'y a plus une vérité, mais des vérités limitées - cette relativité n'ayant rien à voir avec le relativisme ${ }^{10}-$, dès lors qu'il n'y a plus une morale surplombante, mais des constructions singulières/collectives de l'éthique, la question d'un point qui soit « le véritable lieu » ne fait plus sens dans les mêmes termes, pour l'auteur comme pour le lecteur.

Les évolutions contemporaines de la perspective disent l'émergence de la complexité (Morin, 1977, 1980, 2000) et des parcours, tels que les théorise Latour à partir de la notion d'acteur-réseau (Latour, 2006). Le lecteur s'étonnera peut-être de trouver ici des références empruntées à la sociologie et non à la linguistique. C'est qu'une linguistique cantonnée dans les limites de l'analyse de la phrase, est peu armée pour penser la complexité des textes, à la

\footnotetext{
10 Qu'est-ce qui vous fait penser qu' « adopter un point de vue » signifie « être limité »? ou être spécialement « subjectif»? [...] Si vous pouvez avoir différents points de vue sur une statue, c'est parce que la statue ellemême est en trois dimensions et vous permet, oui, vous permet de tourner autour. Si une chose rend possible cette multiplicité de points de vue, c'est qu'elle est très complexe, intriquée, bien organisée, et belle, oui, objectivement belle.

[...] Ne croyez pas à toutes ces foutaises sur le fait d'être «limité » à votre propre perspective. Toutes les sciences ont inventé des moyens pour se déplacer d'un point de vue à un autre, d'un cadre de référence à un autre. [...] C'est ce qu'on appelle la relativité. [...] Si je veux être un scientifique et atteindre à l'objectivité, je dois être capable de naviguer d'un cadre de référence à l'autre, d'un point de vue à l'autre. Sans de tels déplacements, je serais limité pour de bon dans mon point de vue étroit. (Latour, $2006: 210-213$ )
} 
différence de la linguistique textuelle et de l'analyse de discours. Mais le défi d'une saisie globale de la complexité dans des textes décentrés ou polycentrés, à quoi invitent les problématiques conjointes du PDV et de la perspective, requiert sans nul doute des approches conjointes de paradigmes scientifiques différents, notamment des sciences du langage et des approches littéraires, ainsi que Bakhtine l'a montré à travers la notion de dialogisation, qui sert d'interface (Rabatel, 2006) à ses travaux sur la polyphonie et sur le dialogisme.

\section{Références bibliographiques}

Barthes R. (1970) « L’ancienne rhétorique », Communications 16, 172-229.

Bal M. (1977) Narratologies. Klincksieck, Paris.

Banfield A. (1995) Phrases sans parole, Seuil, Paris.

Chartier P. (1990) Introduction aux grandes théories du roman, Bordas, Paris.

Comar P. (1992) La perspective en jeu, Gallimard, Paris.

Damisch H. (1987) L'Origine de la perspective, Flammarion, Paris.

Desportes M. (2005) Paysages en mouvement, Gallimard, Paris.

Charaudeau P. et Maingueneau D. (2002) Dictionnaire d'analyse du discours, Seuil, Paris.

Détrie C., Siblot P., Verine B. (2001) Termes et concepts pour l'analyse du discours, Champion, Paris.

Ducrot O. (1984) Le dire et le dit, Minuit, Paris.

Foucault M. (1966, 2001a) « Entretien avec Madeleine Chapsal », in Dits et écrits 1, 541-546. Gallimard, Paris.

- $(1969,2001 \mathrm{a})$ «Qu'est-ce qu'un auteur? », in Dits et écrits 1, 817-849. Gallimard, Paris.

- (1976, 2001b) «Questions à Michel Foucault sur la géographie », Dits et écrits 2, 28-40.

Gallimard, Paris.

Forest R. (2003) Critique de la raison linguistique, L'Harmattan, Paris.

Genette G. (1972) Figures 3, Seuil, Paris.

- 1983 Nouveau discours sur le récit, Seuil, Paris.

Hamou P. (1995) La vision perspective (1435-1740), Payot, Paris.

Kuno S. (1976) "Subject, Theme, and the Speaker's Empathy. A Reexamination of

Relativisation Phenomenoma", in C. Li (éd) Subjet and topic, Academic press, 419-444.

Latour B. (2006) Changer de société Refaire de la sociologie, La Découverte, Paris.

Lintvelt J. (1981) Essai de typologie narrative, José Corti, Paris.

Morin E. (1977) La méthode 1. La nature de la nature, Seuil, Paris.

- (1980) La méthode 2. La vie de la vie, Seuil, Paris.

- (2000) : Les sept savoirs nécessaires à l'éducation du futur, http://www.agora2\&.org/unesco/7savoirs/

Panofski E. $(1932,1978)$ La perspective comme forme symbolique, Minuit, Paris.

Rabatel A. (1997) Une histoire du point de vue, Klincksieck/CELTED, Paris, Metz.

- (1998) La Construction textuelle du point de vue, Delachaux et Niestlé, Lausanne, Paris.

- (2003) «Le point de vue, entre langue et discours, description et interprétation : état de

l'art et perspectives » Le point de vue. Cahiers de Praxématique, 41, 7-24.

- (2004a) Argumenter en racontant, De Boeck, Bruxelles.

- (2004b) (éd.) Effacement énonciatif et discours représentés, Langages, 156.

- (2005) «Une catégorie transversale, le point de vue », Le français aujourd'hui, 151, Les catégories de la discipline français, 57-68.

- (2006) «La dialogisation au cœur du couple polyphonie/dialogisme chez Bakhtine », Revue romane, 41-1, 55-80.

Raimond M. (1968) La crise du roman, des lendemains du naturalisme aux années 20, José Corti, Paris. 
Reuter Y. (1991) Introduction à l'analyse du roman, Bordas, Paris.

Zribi-Hertz A. (1990) "Lui-même argument et le concept de "pronom A" », Langages, 97, $100-127$. 